\title{
A Critical Review on Removal of Gaseous Pollutants Using Sulfate Radical-based Advanced Oxidation Technologies
}

\author{
Yangxian Liu*, Lei Liu and Yan Wang
}

School of Energy and Power Engineering, Jiangsu University, Zhenjiang, Jiangsu 212013, China

Correspondence author and E-mail: liuyx1984@126.com (Y.X. Liu)

Figures S1-S7 
(a)

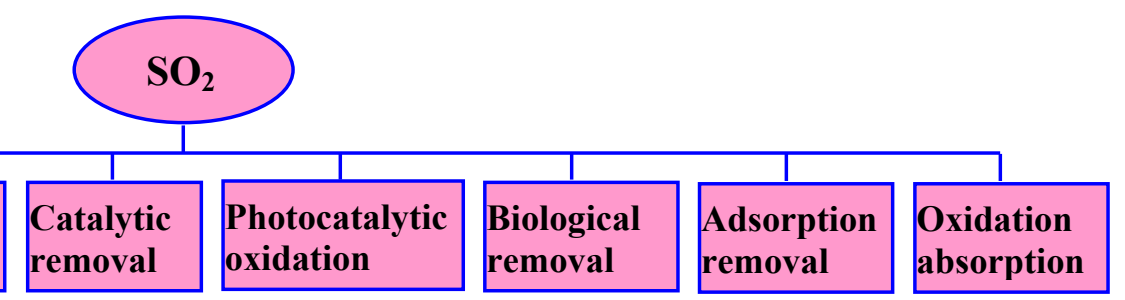

(b)

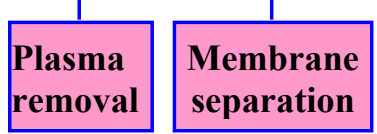

removal oxidation
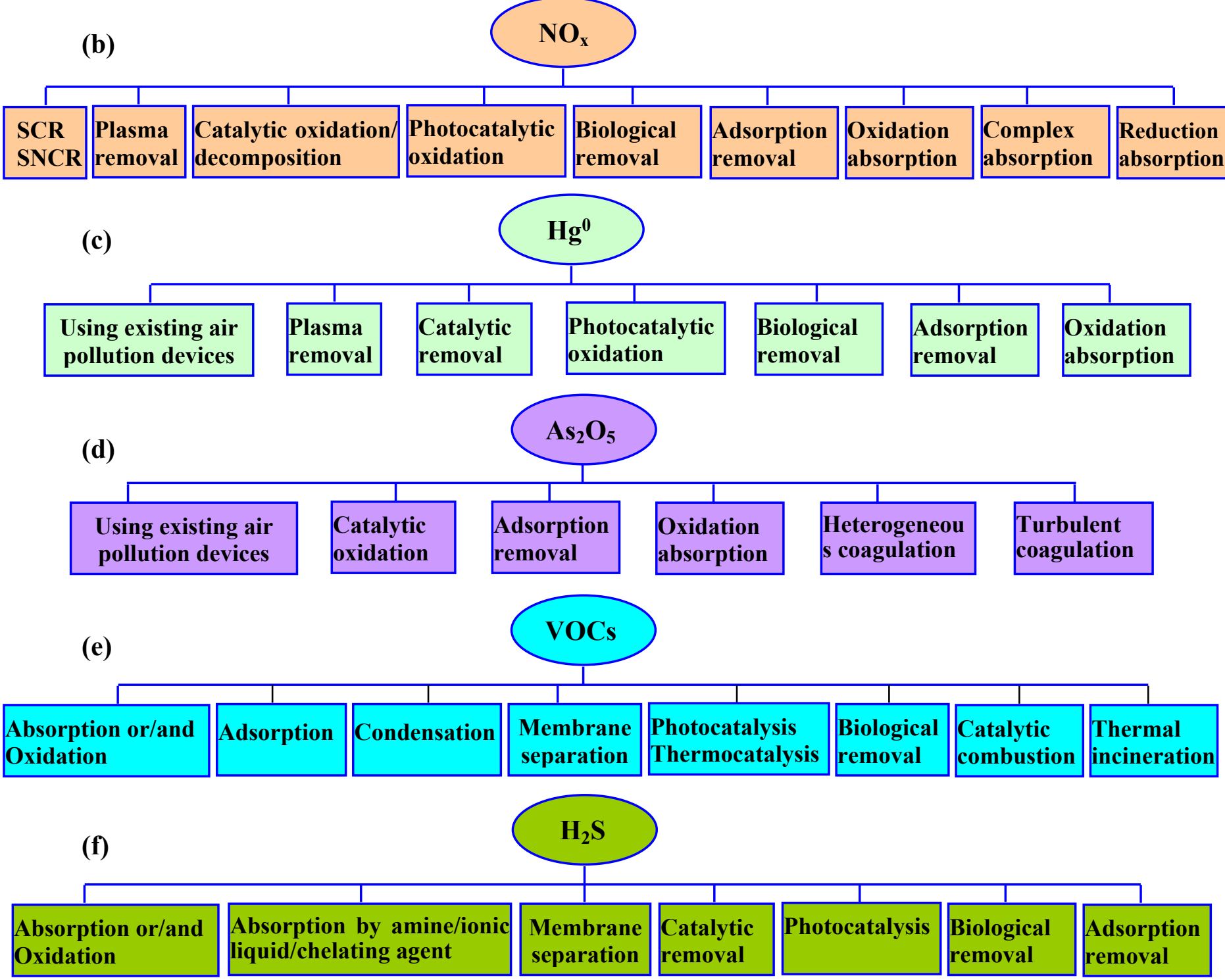

(g)

Simultaneous removal of multi-gaseous pollutants

(two or more)

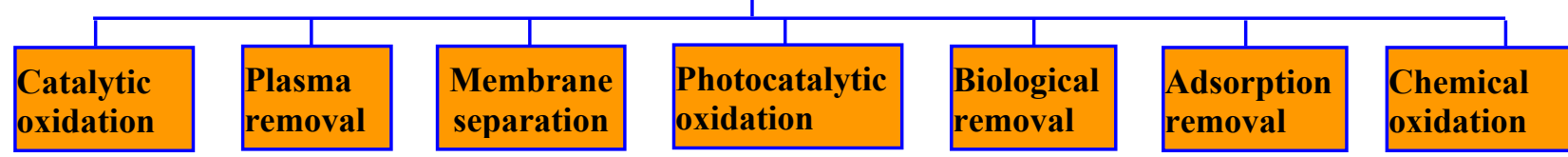

Figure S1. Classification of common gaseous pollutants removal technologies: (a) $\mathrm{SO}_{2}$; (b) $\mathrm{NO}_{\mathrm{x}}$; (c) $\mathrm{Hg}$; (d) As;

(e) VOCs; (f) $\mathrm{H}_{2} \mathrm{~S}$; (g) simultaneous removal of multi-gaseous pollutants. 


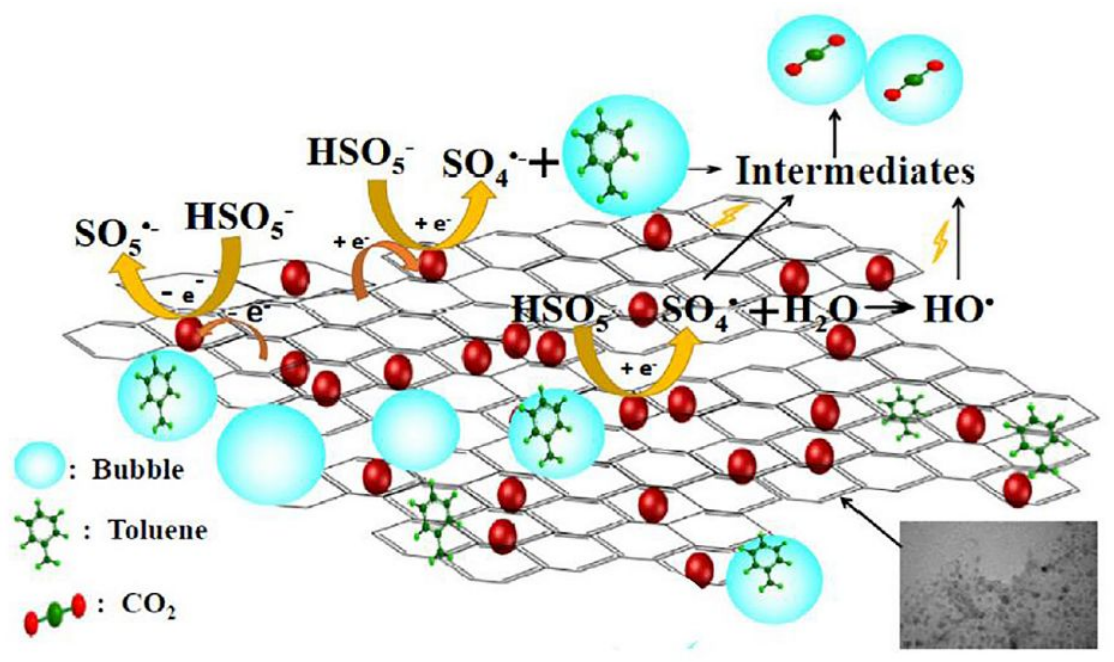

Figure S2. Pathway/mechanism of $\mathrm{Co}_{3} \mathrm{O}_{4} / \mathrm{AC}$ activation of PMS for gaseous toluene degradation in solution

[106]. This figure was reprinted with permission from ref 106. Copyright 2018 Elsevier. 

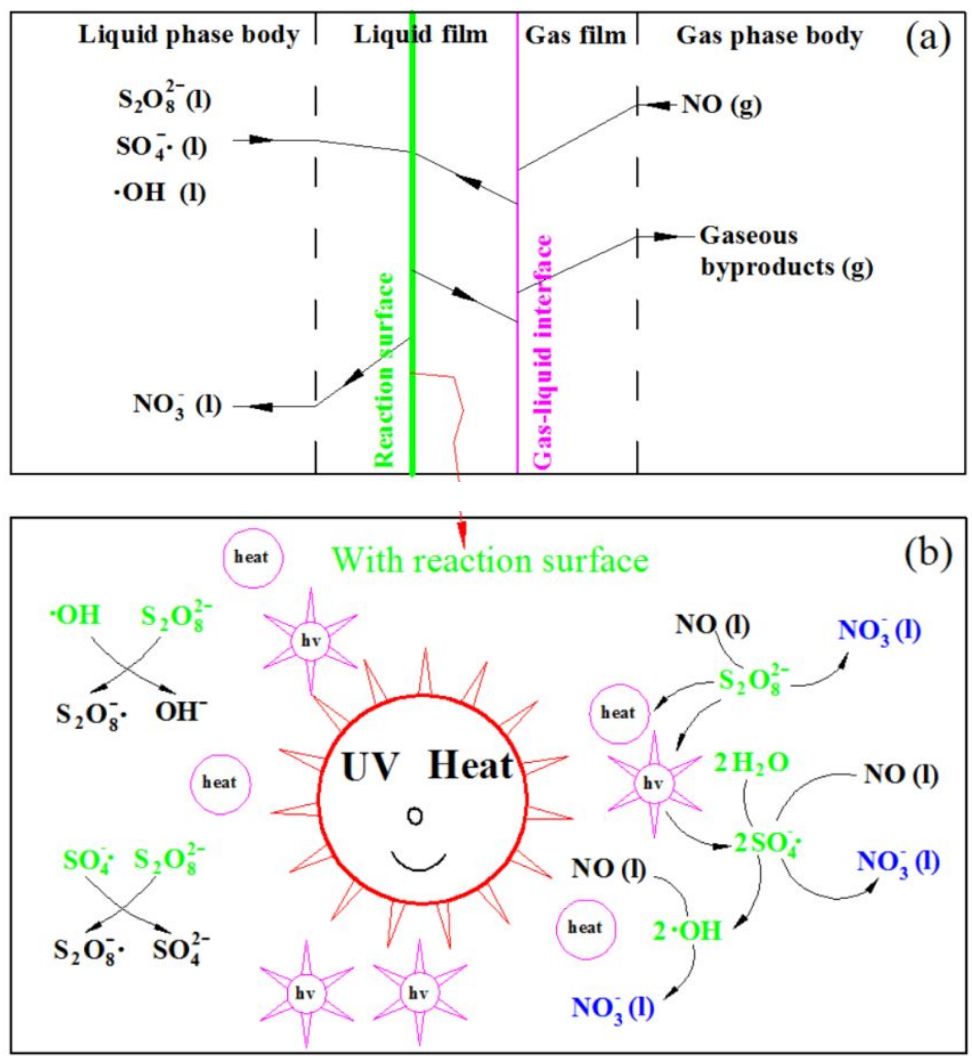

Figure S3. Mass transfer-reaction mechanism of NO removal by $\mathrm{UV} / \mathrm{S}_{2} \mathrm{O}_{8}{ }^{2-}$ in $\mathrm{UV}$-impinging stream reactor

[114]. This figure was reprinted with permission from ref 114. Copyright 2017 Wiley. 


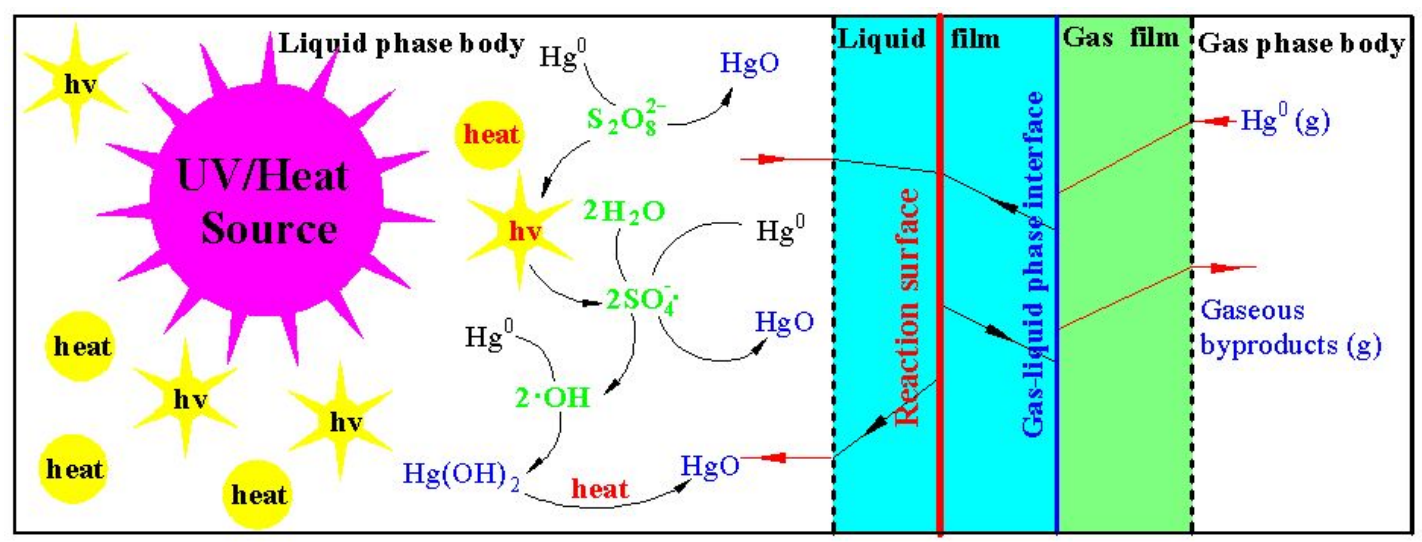

Figure S4. Macroscopic kinetic mechanism of $\mathrm{Hg}^{0}$ removal using UV/heat/PS AOTs [118]. This figure was reprinted with permission from ref 118. Copyright 2018 American Chemical Society. 


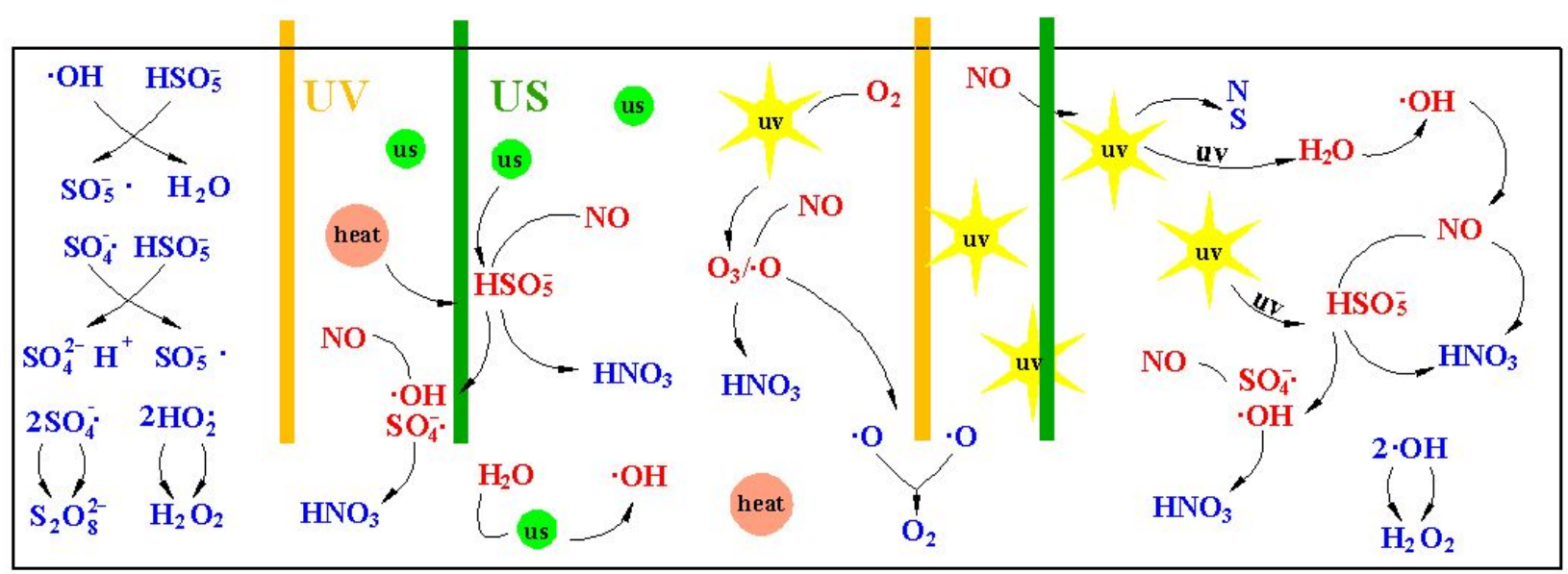

Figure S5. Synergism and mechanism of NO removal by US/VUV/PMS coupled AOT [134]. This figure was reprinted with permission from ref 134. Copyright 2017 American Chemical Society. 

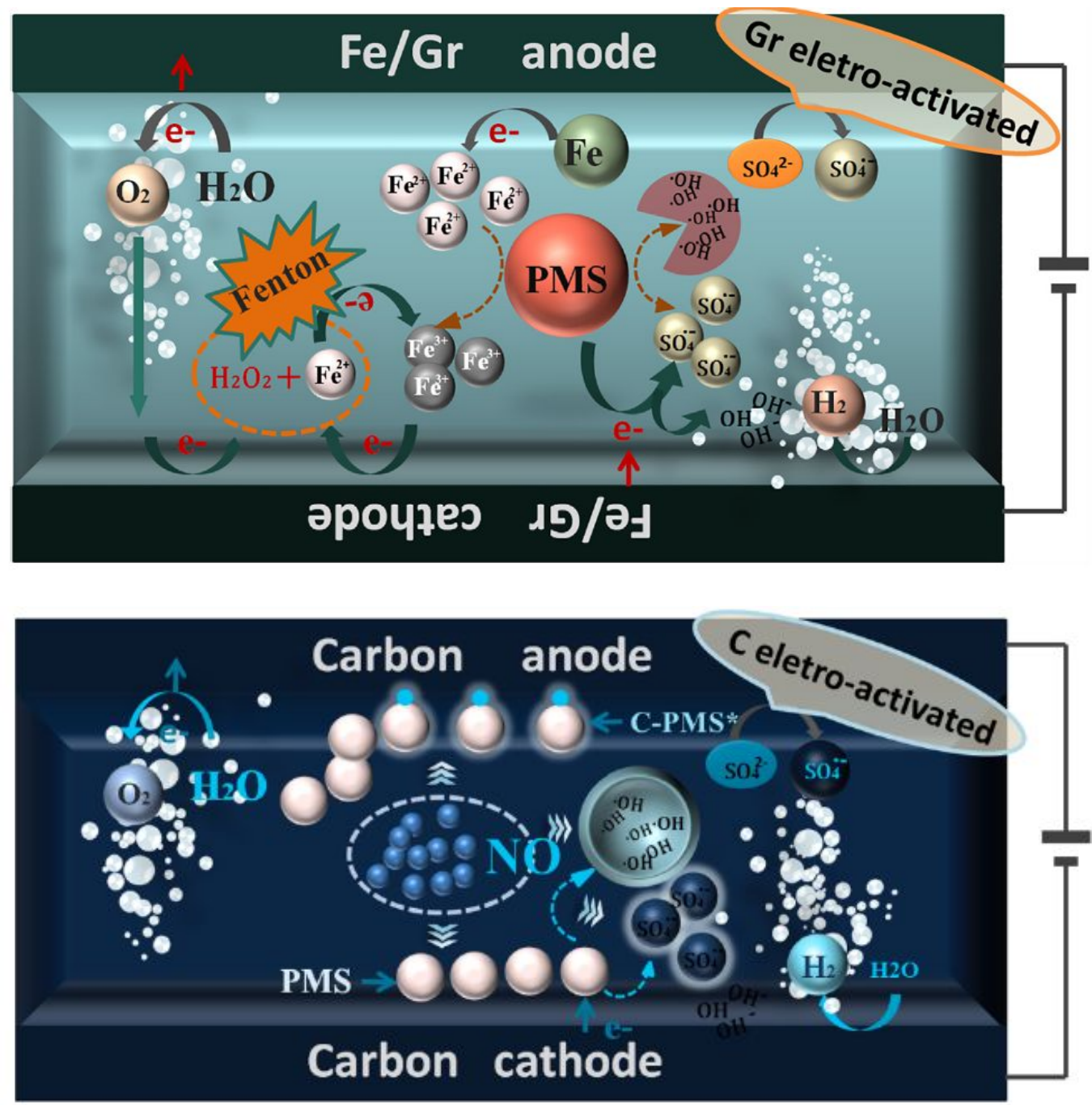

Figure S6. Mechanism of electro-activation of PMS by Fe/Gr [142] (nether) and C/C electrodes (upper) [143].

This figure was reprinted with permission from ref 142 (Copyright 2020 Elsevier) and ref 143 (Copyright 2021 Elsevier). 


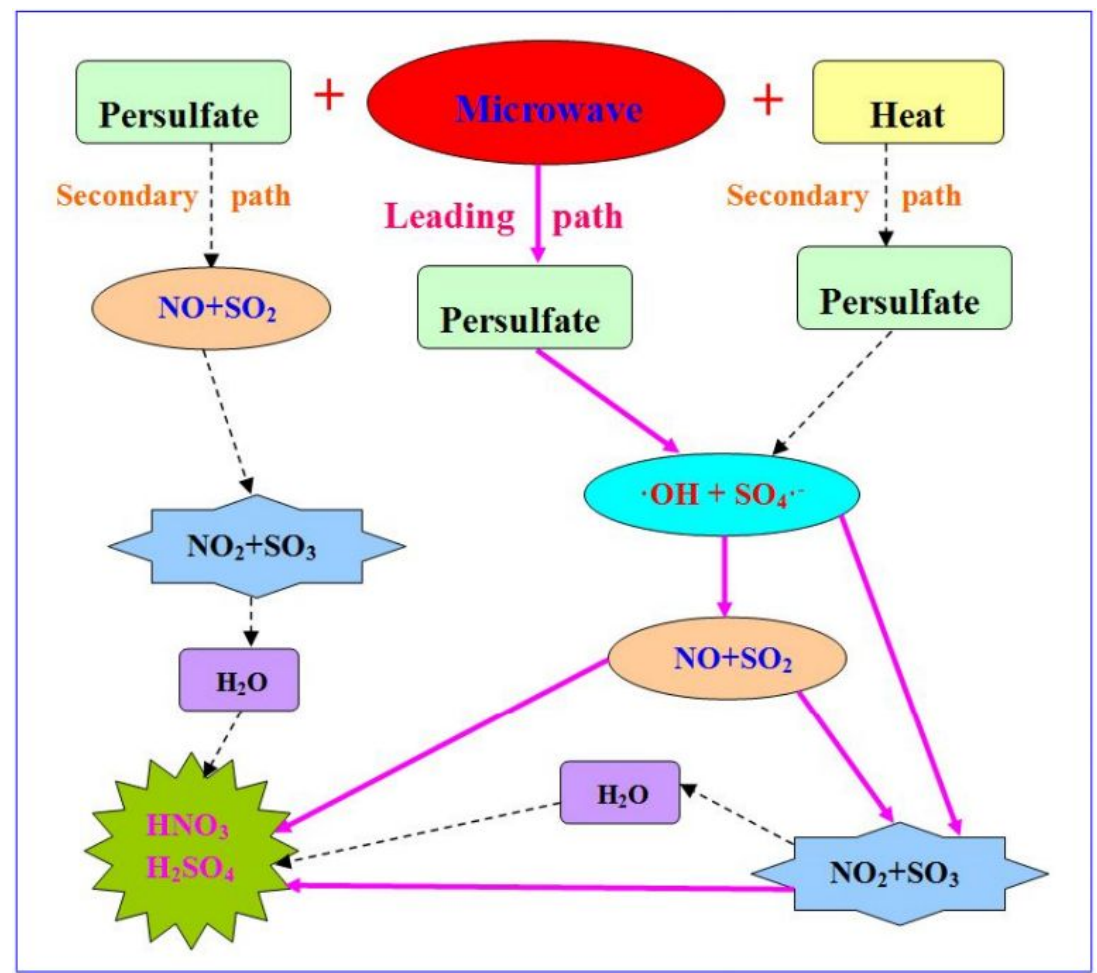

Figure S7. Removal mechanism/pathways of $\mathrm{NO}$ and $\mathrm{SO}_{2}$ using a semi-dry microwave activation technology

[157]. This figure was reprinted with permission from ref 157. Copyright 2020 American Chemical Society. 